\title{
An Update on Myasthenic Crisis
}

Shafiuddin Ahmed, MD

J awad F. Kirmani, MD*

Nazli J anjua, MD

Ammar AlKawi, MD

Ismail Khatri, MD

Abutaher M. Yahia, MD

Nizar Souyah, MD

Adnan I. Qureshi, MD

\author{
Address \\ *Department of Neurology and Neurosciences, University of Medicine and \\ Dentistry of New J ersey, Newark, NJ 07101, USA. \\ E-mail: jkirmani@ hotmail.com
}

Current Treatment Options in Neurology 2005, 7:129-141

Current Science Inc. ISSN 1092-8480

Copyright $\odot 2005$ by Current Science Inc.

\section{Opinion statement}

Myasthenia gravis (MG) is the most common disorder of the neuromuscular junction. Myasthenia crisis, defined as respiratory failure requiring mechanical ventilation in MG, is a common life-threatening complication that occurs in approximately $15 \%$ to $20 \%$ of patients with MG during their life time. The advent of effective mechanical ventilation, specialized neurointensive care units and the widespread use of immunotherapies have substantially altered the prognosis of myasthenic crisis. Early intubation and mechanical ventilation is perhaps the most important step in the management of myasthenic crisis. The authors favor an orotracheal approach for intubation, and placement of small bore duodenal tubes that may help decrease the risk of aspiration and may be more comfortable than regular nasogastric tubes for the patient. Plasma exchange may be more effective than the intravenous immunoglobulin in the treatment of myasthenic crisis involving respiratory failure. A randomized trial is required to confirm the superior efficacy of plasma exchange compared with intravenous immunoglobulin. In the acute setting, the role of immunosuppression and intravenous/ intramuscular pyridostigmine and the newer agents such as tacrolimus remains limited and at times controversial. The therapy should be tailored at an individual basis using best clinical judgment.

\section{Introduction}

Myasthenia gravis (MG) is the most common disorder of the neuromuscular junction. The estimated prevalence in the United States is five to seven (published estimates from 0.5 to 15 ) per 100,000 (or about 40,000 cases) [1, Class III ]. Myasthenic crisis, defined as respiratory failure requiring mechanical ventilation, is a potentially lifethreatening complication that occurs in approximately $15 \%$ to $20 \%$ of patients with MG [1, Class III].

\section{HISTORICAL PERSPECTIVE}

Thomas Willis of Oxford provided perhaps thefirst dinical description of MG in 1672. Approximately 200 years later, Samuel Wilks [2, Class III] described a series of patients with weakness without identifiable brain lesion.

The experience before the 1960s suggested a high mortality of $30 \%$ to $70 \%$ from MG. MG recently has become more treatable with minimal mortal ity (3\% to 
8\%). This has been made possible with the increasing use of cholinesterase inhibitors beginning in the 1930s. Thymectomy became prevalent in 1950s followed by the use of corticosteroids and other immunosuppressive agents, which gained wide acceptance in the 1960s.

Plasma exchange (PE) in the mid-1970s and intravenous immunoglobulin (IVIg) and new immunosuppressant (in the 1980s and 1990s) eventually were introduced for treatment of MG. Use of neurointensive care units in the 1990s dramatical ly hel ped with reduction of mortal ity. With the advent of effective mechanical ventilation in the 1960s, greater improvement in morbidity and mortality rates of patients with MG was seen [3, Class III].

\section{EPIDEMIOLOGY}

The reported preval ence of myasthenic crisis increased from the 1950s to the 1990s [4, Class III]. This largely reflects the increased life span of patients with MG because of improved treatment. MG prevalence is higher in women under the age of 50 years than in men of the same age. For men older than 50 years of age, the prevalence of MG is higher than in women. African-Americans are affected more by this disease in all age ranges [5, Class III]. With an increase of the number of older patients in population demographics, the number of patients older than age 50 years may be twice the number of those younger than age 50 years by the year 2005 .

There are estimated 40,000 cases of MG in the US; approximately $20 \%$ (8000 cases) are expected to have a very benign ocular $M G$, and a similar number of patients are expected to experience at least one life-threatening myasthenic crisis in their lifetime.

\section{CLINICAL FEATURES}

The weakness in MG characteristically becomes worse with repeated activity. Signs of weakness are usually first evident in the eyelids and extraocular muscles, and generalized weakness follows in $85 \%$ of cases [4, Class III]. The proximal limb muscles often are affected, and weakness can involve the diaphragm and thoracic musculature. The deficit in myasthenia is purely motor. Deep tendon reflexes usually are preserved.

Myasthenic crisis is defined by an acute episode of respiratory muscle weakness (defined by vital capacity
[VC] of $1 \mathrm{~L}$ or less, negative inspiratory force [NIF] 20 $\mathrm{cm} \mathrm{H}_{2} \mathrm{O}$ or requirement of mechanical ventilation). Rapid progression to respiratory failure in MG can occur in patients with recent worsening of symptoms, especially if the oropharyngeal muscles are involved. The crisis could manifest as oropharyngeal bulbar crisis where the upper airway is compromi sed or it could also be the respiratory failure secondary to muscle weakness. Respiratory support usually is required [6, Class III]. The advent of special ized neurointensive care units and the widespread use of immunotherapies have substantially al tered the management of myasthenic crisis.

\section{CONDITIONS MIMICKING CRISIS}

In cases of excessive acute or chronic overdose of anticholinesterase medications, muscle weakness mimicking myasthenic crisis may develop. This is caused by excess of acetylcholine (ACh) at the motor effector sites. The possibility that the deterioration could becaused by cholinergic crisis is best excluded by temporarily stopping anticholinesterase drugs while the patient is presumptively treated for the myasthenic crisis. Acute treatment in such a setting is supportive does not require any specific or different intervention for myasthenic versus cholinergic crisis.

\section{DIAGNOSTIC TESTING}

A quick bedside technique for diagnosing $M G$ is the ice test. Improvement of the ptosis after putting the ice on the eyelid for 2 minutes, suggests a disorder of neuromuscular transmission [7,8, Class III].

Laboratory studies The most sensitive and specific test for MG is the presence of acetylcholine receptor antibodies (AchR-Ab), it can beconfirmed dinically by edrophonium (Tensilion) administration and by el ectrodiagnostics. Musde-spedific receptor tyrosine Kinase (MuSK) [9, Class III] is most useful when testing patients who have MG but are AchR-Ab negative

Radiographic studies Approximatel y $20 \%$ of patients with MG have a thymoma present, whereas approximately $70 \%$ have thymic hyperplasia [10, Class III ]. All patients with MG should have a CT scan of chest done with contrast.

\section{Treatment}

- The al gorithm for the treatment of myasthenic crisis consists of an approach that includes: initiation of supportive care, elimination of possible other conditions that may mimic crisis, removal of trigger or exacerbating factors, and treatment of underlying MG.

- Management of myasthenic crisis should be done in an intensive care unit staffed with physicians who are experienced in themanagement of the disease itself, respiratory insufficiency, infectious disease, and fluid and electrolyte 


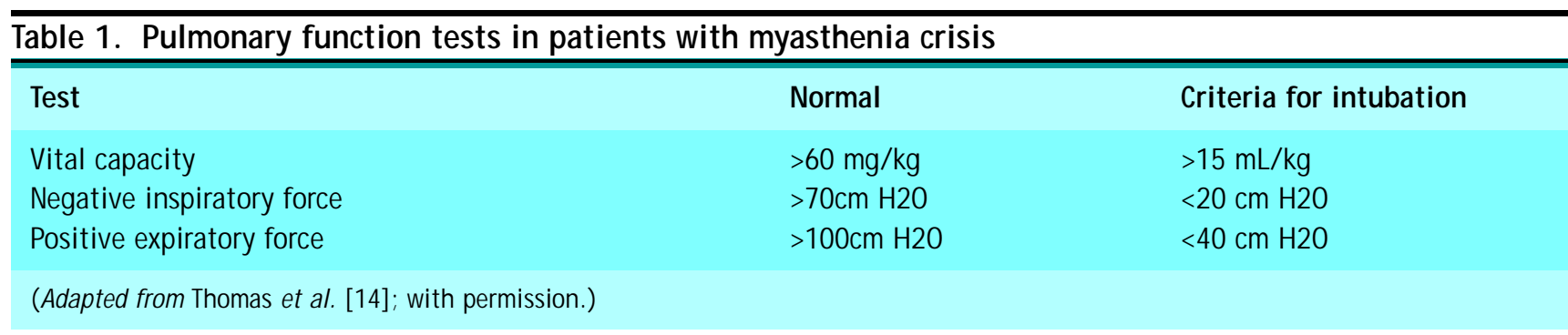

balances. An experienced neurointensivist provides an optimal patient care In the absence of the expertise of the neurointensivist, a team of neurologist and critical care physicians is required. After stabilizing the patient and securing the airway, respiration, and hemodynamics, options for specific treatment indude use of acetylcholinesterase (AChE) inhibitors, PE, IVIg, and immunosuppressive drugs. The prognosis has improved strikingly as a result of advances in treatment; virtual ly all patients with myasthenic crisis can be returned to full productivelives with proper therapy [11, Class III].

- The patient should be stabilized at the time of presentation. After securing the airway, respiration, and circulation, usually in the emergency room, the patient is transferred to an intensive care setting.

- Thereare intensive care issues that are specific to the management of theMG. Unlike most other patients that are dose to respiratory failure, patients with MG, especiall ly younger patients, areable to maintain good oxygen saturation. The patient suspected of going into crisis should have monitoring of $\mathrm{VC}$ and NIF every hour. Clinical state, use of accessory muscles, and subjective feeling of air hunger are other reliable indicators of impending respiratory failure The authors recommend liberal intubation of patients with MG; early intubation and mechanical ventilation is perhaps the most important step in the management of myasthenic crisis [1,12•, Class III].

- The authors base the dedision to intubate on the dinical evaluation that indudes the assessment of strength of cough and sniff. Patients are asked to count loudly as long as possiblein one breath. Normally, onecan count easily up to 30, whereas the patients who count less than 20 have significant impairment of respiratory musdes. Consider intubation if a patient with myasthenic crisis has the following pulmonary function tests [12•, Class III] (Table 1).

- The authors favor an orotracheal approach for intubation; a soft low-pressure cuff is recommended. Tracheostomy generally is unnecessary, because the duration of intubation is frequently less than 2 weeks [13,14, Class III]. After the patient is placed on a respirator, synchronized intermittent mandatory ventilation with minimal sedation is recommended for the initial setting. Relatively larger tidal volumes, 8 to $10 \mathrm{~mL} / \mathrm{kg}$ with pressure support of 8 to 15 $\mathrm{cm} \mathrm{H} 2 \mathrm{O}$ areencouraged. This prevents the devel opment of atel ectasis. Caution should be used to keep peak airway pressures less than $45 \mathrm{~cm}$ of $\mathrm{H} 2 \mathrm{O}$. The adequacy of ventilatory support is determined by the patient comfort and lack of $\mathrm{CO} 2$ retention. Supplemental oxygen above $30 \%$ FiO2 is rarely necessary. The authors recommend keeping theinspired humidity of $60 \%$ to $90 \%$, depending on the amount of secretions. The patient then is observed for usual ventilator care and frequent checks of cuff pressure, tube placement, and blood gases. Frequent aseptic deep suctioning, preferably with a soft suctioning catheter, is helpful in avoiding complications [15•, Class III]. 
- Weaning attempts in the patients with myasthenic crisis should al ways begin when: 1) Overall strength is improving; 2) VC exceeds $10 \mathrm{~mL} / \mathrm{kg} ; 3$ ) $\mathrm{NIF}$ negativel y exceeds $-20 \mathrm{~cm} \mathrm{H}_{2} \mathrm{O}$; 4) oxygenation is normal (FiO2 40\%); and 5) the patient is free of infection or other medical complications. The trial should start early in the day initially with continuous peak airway pressure and high-pressure support. After weaning the rate to 0 , it usual ly is helpful early on to set the pressure support to 8 to $15 \mathrm{~cm} \mathrm{H}_{2} \mathrm{O}$. If the patient is able to tolerate this for at least 1 day, pressure support is decreased by 1 to $2 \mathrm{~cm} \mathrm{H}_{2} \mathrm{O}$ to at least $5 \mathrm{~cm} \mathrm{H}_{2} \mathrm{O}$ (just enough to overcome the circuit resistance). If the results of previous assessments reveal fatigue with tachypnea, patients are rested again on synchronized intermittent mandatory ventilation with a set rate of at least 6 [16••, Class III].

- As in any other critically ill patients, negativeenergy balancewill interferewith weaning efforts, an early start of appropriate nutrition is essential. This becomes even more important in patients with MG, because of the relative borderline strength of respiratory musdes. Clinicians should remember that patients in myasthenic crisis are not being ventilated because of pulmonary or systemic problems, but because of the problems with muscle strength. The authors favor early placement of small bore tubes that end in the duodenum that may help decrease the risk of aspiration and may be more comfortable than a regular nasogastric tubes for the patient [12•, Class III].

- In addition to the specific measures mentioned, general intensive care monitoring should beoptimized with aggressive treatment of other systems. Hypokalemia, hypomagnesemia, and hypophosphatemia that may exacerbate weakness should betreated aggressively. Anemia will affect the oxygen carrying capacity and also may additionally exacerbateweakness; therefore, transfusions for a hematocit value of $30 \%$ and bel ow are recommended. Deep venous thrombosis prophylaxis with the use of subcutaneous heparin or the use of sequential compression devices is necessary.

\section{Identification and elimination of exacerbating factors}

- In addition to concurrent illness and surgery, several drugs can precipitate or aggravate the MG into myasthenic crisis. The principal offenders are antibiotics (aminoglycosides), cardiac drugs (lidocaine, procainamide, or quinidine), and magnesium [17, Class III]. Aminoglycosides can precipitatea myasthenic syndrome that resolves when the drugs are discontinued. Magnesium blocks the presynaptic release of ACh and can be particularly detrimental in patients with myasthenic crisis (Table2) [14, Class III].

- The most common cause of crisis is concurrent infection. This should be treated immediately, because the mechanical and immunologic defenses of the patient are compromised. The patient with MG with fever and early infection should be treated like any other patient who is immunocompromised. Early and effective antibiotic therapy, respiratory assistance, and pulmonary physiotherapy are essential to the treatment regimen. As will be discussed later, PE and IVIg frequently are hel pful in hastening recovery.

Pharmacologic treatment

- The immediate pharmacologic treatment of myasthenic crisis includes use of anticholinesterase medications, PE, or IVIg. Long-term immunosuppression with steroids beginning in the acute phase and extending into intermediate periods of days to weeks may be hel pful. All other forms of immunosuppression usually are reserved for the longer-term and usually are not initiated or 


\begin{tabular}{lcc}
\hline Table 2. The experience of $\mathbf{7 3}$ patients with myasthenic crisis \\
\hline & $\begin{array}{c}\text { Primary participant, } \\
\text { n(\%) }\end{array}$ & $\begin{array}{c}\text { Possible contributing factor, } \\
\mathbf{n}(\%)\end{array}$ \\
& $28(38)$ & $6(8)$ \\
Infection & $12(16)$ & $2(2)$ \\
Bacterial pneumonia & $6(8)$ & $0(0)$ \\
Viral URI & $4(5)$ & $1(1)$ \\
Bacterial URI & $3(4)$ & $1(1)$ \\
Sepsis & $3(4)$ & $2(2)$ \\
Other & $22(30)$ & - \\
No obvious precipitant & $7(10)$ & $7(10)$ \\
Aspiration pneumonitis & $6(8)$ & $2(2)$ \\
Medication-related & $2(2)$ & $0(0)$ \\
Steroid administration & $2(2)$ & $1(1)$ \\
Steroid withdrawal & $1(1)$ & $1(1)$ \\
Aminoglycoside use & $3(4)$ & $3(4)$ \\
Pregnancy/ postpartum & $3(4)$ & $3(4)$ \\
Upper airway obstruction & $2(2)$ & $0(0)$ \\
Surgery & $2(2)$ & $15(21)$ \\
Other & & \\
URI- upper respiratory infection. & \\
(Adapted from Thomas et al. [14]; with permission.) &
\end{tabular}

maintained during acute crisis. The other modalities of immunosuppression are briefly discussed for the sake of completeness because many patients with MG are chronically treated with it when they present with crisis.

\section{Anticholinesterase medications}

- There is no substantial difference in efficacy among the various anticholinesterase drugs; oral pyridostigmineis the onemost widely used in theUS. Asa rule, the beneficial action of oral pyridostigmine begins within 15 to 30 minutes and lasts for 3 to 4 hours, but individual responses vary in patients with myasthenic crisis.

- In case of myasthenic crisis, the following also should be considered when using anticholinesterase medications:

- Overdosage with AChE inhibitors may exacerbate weakness. These agents al so makeit more difficult to assess responseto other therapies, and the authors think that a period of withdrawal may increase response to these medications. For these reasons, the authors recommend stopping AChE inhibitors for patients in myasthenic crisis and re-instituting them orally when patients are getting stronger and are about to be extubated [18•,19,20, Class III]. The authors restart the medications at a low dosage, and gradually increase to a dosage that produces dear benefit. Pyridostigmineal so has been shown to increase sensitivity to non-depolarizing anesthetics such as vecuronium, which may be potentially hazardous in patients planned for thymectomy [21, Class I].

- An alternativeapproach is to useintravenous pyridostigmineinfusion at 1 to $2 \mathrm{mg}$ per hour during arisis. Each milligram of intravenous pyridostigmine is equival ent to $30 \mathrm{mg}$ of oral pyridostigmine (some authorities think it is as low as 8 to $10 \mathrm{mg}$ ), and is infused with nomal saline In one small retrospectivestudy [13, Class III], crisis patients receiving intravenous pyridostigmine showed dinical improvement comparable with those receiving PE. However, some patients have 
experienced significant, sometimes fatal, cardiac armythmias while receiving intravenous pyridostigmine [13,18•, Class III]. To reduce side effects, oneapproach is to administer intramuscular pyridostigminethat may be equally effective, but may reducethe risk of cardiac arrhythmias. Furthermore, AChE inhibitors can promote excessive secretions, which block feeding or endotracheal tubes, cause mucus plugging and lead to atelectasis [12•, Class III].

- In most settings, it may become difficult to differentiate between weakness caused by excessive AChE and myasthenic crisis as explained; therefore, the authors consider stopping the AChE drugs at least until early improvement is detected clinically.

- Long-acting pyridostigmine tabl ets may help to improve patient's symptoms through the night, but because of variable absorption should never be used for myasthenic crisis.

Pyridostigmine bromide

Standard dosage The average dose of oral pyridostigmine is 15 to $60 \mathrm{mg}$ tablets or $105-\mathrm{mL}$ teaspoonfuls daily, which are spaced to provide maximum relief.

Contraindications Pyridostigmine is contraindicated in patients with mechanical intestinal or urinary obstruction.

Main drug interactions Corticosteroids and quinidine reduce the effect of pyridostigmine. The effect of succinylcholine is moderately increased in the presence of pyridostigmine. Atropine antagonizes the muscarinic effects of pyridostigmine, and this interaction is used to counteract the muscarinic symptoms of pyridostigmine toxicity.

Main side effects Diarrhea, abdominal cramps, salivation, and nausea and vomiting.

Special points The muscarinic side effects of the AChE medication may limit the dose tolerated by some patients. In these cases, propantheline bromide may be used to block the autonomic side effects without altering the beneficial effects on skeletal muscle. Loperamide also is useful for the treatment of diarrhea induced by AchE medications.

cost/ cost effectiveness $60 \mathrm{mg}$ three times daily costs $\$ 60$ to $\$ 100$ per month.

Plasma exchange has been used therapeutically in patients with myasthenic crisis. Plasma, which contains the pathogenic antibodies, is mechanically separated from other blood components, which are returned to the patient.

The retrospective multicenter analysis done by the authors' group suggests that the $\mathrm{PE}$ is more effective than the IVIg in the treatment of myasthenic crisis involving respiratory failure. However, of 54 episodes of myasthenic crisis, more patients had complications related to PE than IVIg [22•, Class III ]. An analysis of the ventilatory status at 2 weeks $(P=0.02)$ and at 1 month $(P=0.04)$ revealed a higher rate of successful extubation in the group treated with PE. A randomized trial is required to confirm the superior efficacy of PE compared with IVIg.

Gajdos et al. [23•, Class III] did not find any differences in efficacy and tolerance of two regimens of IVIg (3- and 5-day courses of $400 \mathrm{mg} / \mathrm{kg}$ per day) compared with three cycles of PE in patients presenting with MG exacerbation that included, but was not restricted to, respiratory failure. The difference between results of the authors' study and the previous study may be attributed to higher frequency of mechanically ventilated patients ( 42 of 54 versus nine of 87 episodes), and larger number of exchanges done per person in the authors' study. In contrast, Stricker et al. [24, Class III] reported four patients with myasthenic crisis who initially were treated with IVIg without any clinical improvement. All four patients improved with subsequent PE.

Standard procedure A course of five exchanges ( 3 to $4 \mathrm{~L}$ per exchange) generally is administered over an alternate day regimen for 10 days [25•, Class III].

Contraindications Contraindications related to extracorporeal lines and anticoagulation include precarious hemodynamics, unstable angina pectoris, and active internal bleeding. Contraindication related to immunosuppression is mainly concern for sepsis and septic shock. 
Complications Immediate complications usually are related to extracorporeal lines, anticoagulation, and replacement fluids, and include excessive bleeding, pneumothorax, and exacerbation of underlying congestive heart failure. Delayed complications include hypercoagulable state and predisposition to infections secondary to immunosuppressed state.

Special points Plasma exchange produces a short-term reduction in anti-AchR-ab, with clinical improvement in many patients. It is useful as a temporary expedient in patients with myasthenic crisis or to improve the patient's condition before surgery (thymectomy).

cost/ cost effectiveness The approximate cost for each PE is $\$ 1500$.

Intravenous immunoglobulin

The indications for the use of IVIg are the same as those for PE. It produces rapid improvement to help the patient through a difficult period of myasthenic crisis or before surgery.

This treatment has the advantages of not requiring special equipment or largebore venous access. Although there have been no direct trials comparing PE with IVIg, the latter has been compared with placebo in a small double-blinded random, controlled trial. Among 15 patients, Wolfe et al. [26, Class II] found no difference at 42 days but some trend towards improvement at 6 months in patients receiving $2 \mathrm{gm} / \mathrm{kg}$ of IVI g. Improvement occurs in about $70 \%$ of patients, beginning during treatment, or within 4 to 5 days thereafter, and continuing for weeks to months.

Standard dosage The usual dose is $2 \mathrm{~g} / \mathrm{kg}$, which typically is administered over 5 days ( $400 \mathrm{mg} / \mathrm{kg}$ per day). If tolerated, the course of I VIg can be shortened to administer the entire dose over a 3-day period.

Contraindications This treatment should not be given to patients with isolated immunoglobulin $A$ (IgA) deficiency. Such individuals have the potential for developing antibodies to IgA and could have anaphylactic reactions to subsequent administration of blood products that contain IgA.

Main drug interactions Antibodies present in immune globulin may interfere with the immune response to certain live viral vaccines including measles, mumps, rubella, and varicella vaccines.

Main side effects Adverse reactions are uncommon, but include headache, fluid overload, and rarely renal shutdown [22•, Class III].

Special points The mechanism of action of IVIg is not known; the treatment has no consistent effect on the measurable amount of circulating AchR-Ab.

Cost/ cost effectiveness The cost for an adult course is between $\$ 8000$ and $\$ 12,000$, depending on the amount and number of doses used.

\section{Immunosuppression}

- Immunosuppression using glucocorticoids, azathioprine, and other drugs is effective in nearly all patients with myasthenic crisis. The choice of drugs or other immunomodulatory treatments should be guided by the relative benefits and risks for each individual patient and the urgency of treatment. It is hel pful to devel op a treatment plan based on short-term, intermediateterm, and long-term objectives. Immediate improvement is essential because of the severity of weakness in myasthenic crisis; therefore, plasmapheresis should be undertaken or IVIg should be administered. For the intermediate-term, glucocorticoids and cyclosporine general ly produce clinical improvement within a period of 1 to 3 months. The beneficial effects of azathioprine and mycophenolate mofetil usually begin after many months (up to a year); these drugs have advantages for the long-term treatment of patients with MG.

- Longer-term treatment strategy with various immunosuppressive agents, including glucocorticoids, should be considered as the initial improvement from the time myasthenic crisis is observed. Side effects of each drug may preclude its use in certain patients. 
- Corticosteroids are relatively contraindicated in patients with myasthenic crisis with severe diabetes, ongoing infections, or severe osteoporosis. Tuberculosis should be exd uded before starting corticosteroid therapy or other forms of chronic immunosuppression.

- To evaluate the effectiveness of treatment and assess the drug-induced side effects, it is important to have an idea of patient's clinical status at baseline and on repeated interval examinations in a systematic manner. The most useful dinical tests include forward arm abduction time (up to a full 5 minutes), forced VC, range of eye movements, and time for development of ptosis on upward gaze. Manual muscle testing also is important. A progressive reduction in the patient's AChR antibody level also provides serologic valuable confirmation of the effectiveness of treatment.

\section{Glucocorticoid therapy}

- Glucocorticoids, when used properly, produce improvement in myasthenic weakness in the majority of patients [17, Class III]. Unfortunately, relief may take weeks to become optimal. Furthermore, $1 / 3$ of the patients have some exacerbation of their symptoms during the first few days of therapy [17, Class III]. Therefore, the initial dose of prednisone should be relatively low ( 15 to $25 \mathrm{mg}$ per day) in patients with myasthenic crisis, especially if the patient is not intubated.

- The authors prefer to start prednisone when the patient is still intubated and maintained on the ventilator, so that any early exacerbation can be managed appropriately. Maximum dose is initiated on patients who are intubated; otherwise the dose is increased in a stepwise fashion, as tolerated by the patient (usually by $5 \mathrm{mg}$ per day at 2- to 3-day intervals), until there is marked clinical improvement or a dose of $100 \mathrm{mg}$ per day is reached. Some authors have disputed the early exacerbation on initiation of steroids, especially in the setting of concomitant use of PE or IVIg. Therefore, they advocate high-dose intravenous steroids in this setting to hasten the intermediate-term benefit of the steroids.

- The maximum dose is maintained for 1 to 3 months and then is gradually modified to an alternate-day regimen over the course of an additional 1 to 3 months; the goal is to reduce the dose to zero or to a minimal level on the "off day." Generally, patients begin to improve within a few weeks after reaching the maximum dose, and improvement continues to progress for months or years. 
Peptic ulcer may develop with possible perforation and hemorrhage. I mpaired wound healing and may suppress reactions to skin tests. Prednisone also may cause increased intracranial pressure with papilledema (pseudotumor cerebri) usually after treatment, convulsions, vertigo, and headache.

Prednisone also causes menstrual irregularities, development of Cushingoid state, secondary adrenocortical and pituitary unresponsiveness; decreased carbohydrate tolerance; manifestations of latent diabetes mellitus; increased requirements for insulin or oral hypoglycemic agents in patients with diabetes.

Special points Corticosteroids may mask some signs of infection, and new infections may appear during their use.

Cost/ cost effectiveness Prednisone therapy, even in high doses, does not cost more than $\$ 20$ per month.

Azathioprine

Azathioprine has been the most widely used agent because of its relative safety in most patients and long track record. Its therapeutic effect may add to that of glucocorticoids or allow the reduction of the steroid dose.

Standard dosage 3 to $5 \mathrm{mg} / \mathrm{kg}$ daily.

Contraindications Azathioprine should not be given to patients who have shown hypersensitivity to the drug or those who are pregnant.

Main drug interactions Allopurinol increases the levels of azathioprine. A combination of angiotensinconverting enzyme inhibitors with azathioprine increases the adverse effect profiles of each drug.

Main side effects Leukopenia, liver function abnormality, increased risk of malignancy [27, Class III ], nausea, vomiting, diarrhea, fever, malaise, and myalgias.

Special points Azathioprine takes at least 6 weeks to start to show effect and months or even longer to peak, and it is seldom initiated during the treatment of myasthenic crisis.

cost/ cost effectiveness One month of azathioprine therapy costs approximately $\$ 50$.

Cyclosporine

Cyclosporine may be as effective as azathioprine and is being used increasingly in the management of myasthenic crisis. I ts beneficial effects seem similar to those of azathioprine. Therefore, it may be a useful adjunct to glucocorticoids for the intermediate phases of improvement of myasthenic crisis.

Standard dosage 3 to $8 \mathrm{mg} / \mathrm{kg}$ per day. The authors usually administer the drug in two divided doses to minimize the side effects.

Contraindications In patients with a hypersensitivity to cyclosporine and polyoxyethylated castor oil.

Main drug interactions The use of bosentan with cyclosporine is contraindicated, because it may dramatically increase the effect of bosentan and decrease cyclosporine levels. The use of tacrolimus increases the adverse event profile of the drugs. The other drugs that should be avoided in combination include amphotericin B, barbiturates, hydantoins, macrolide antibiotics, digoxin, and carbamazepine.

Main side effects The primary adverse reactions of cyclosporine therapy are renal dysfunction, tremor, hirsutism, hypertension, chronic renal failure, and gum hyperplasia.

Special points It may be used alone, but usually is used as an adjunct to glucocorticoids to permit reduction of the steroid dose.

Cost/ cost effectiveness One month of cyclosporine therapy costs approximately $\$ 300$.

Mycophenolate mofetil

Mycophenolate mofetil, which has been used for immunosuppression in transplant patients, proved useful in the treatment of MG [28, Class I]. It has been shown to improve single fiber EMG findings of "jitter" in a randomized controlled trial $[29$, Class I].

The advantage of mycophenolate is in its relative lack of adverse side effects.

Standard dosage 0.5 to $1.5 \mathrm{~g}$ twice per day orally.

Contraindications Mycophenolate mofetil is contraindicated in patients with hypersensitivity to mycophenolate mofetil, mycophenolic acid, or any component of the drug product. 
Main drug interactions The effect of mycophenolate is significantly decreased in combination with cholestyramine, magnesium hydroxides, and aluminum hydroxides, and should be avoided in combination.

Main side effects Adverse reactions associated with the administration of mycophenolate mofetil include diarrhea, leukopenia, sepsis, and vomiting.

Special points This drug may become preferred for long-term treatment of patients with myasthenic crisis. Unfortunately, it has minimal use for the acute setting.

Cost/ cost effectiveness Mycophenolate therapy for 1 month costs approximately $\$ 500$ ranging from $\$ 250$ to $\$ 800$ depending on the dosage used.

Cyclophosphamide

Cyclophosphamide is reserved for patients who are refractory to the other drugs, because of its relatively high risk of adverse side effects, including late development of malignancies. It has been shown in a double-blinded, random, controlled trial to decrease overall steroid requirements when used chronically in patients with severe MG [30, Class I].

Standard dosage Oral dosing is usually in the range of 1 to $5 \mathrm{mg} / \mathrm{kg}$ per day.

Contraindications Cyclophosphamide is contraindicated in patients who have demonstrated a previous hypersensitivity.

Main drug interactions The combination of succinylcholine and digoxin should be avoided with cyclophosphamide because of interactions increasing the effect of succinylcholine and decreasing the effect of digoxin when used with cyclophosphamide.

Main side effects Hemorrhagic cystitis, pneumonitis, polyneuropathy, cardiomyopathy, and alopecia. Malignancies have developed in patients treated with cyclophosphamide.

Special points This drug also has minimal use in the acute or intermediate setting.

Cost/ cost effectiveness Cyclophosphamide therapy for 1 month costs approximately $\$ 150$.

FK 506 (Tacrolimus)

FK 506 should be considered as an alternative treatment for patients with disabling MG that is not responsive to conventional immunosuppressants, or when other agents are contraindicated. It may be of particular benefit in patients whose sera test positive for anti-Ryanodine receptors [31, Class III].

Standard dosage Oral dosage is recommended in the range of 0.1 to $0.15 \mathrm{mg} / \mathrm{kg}$ daily divided every 12 hours. The intravenous infusion could be given at 0.03 to $0.05 \mathrm{mg} / \mathrm{kg} / \mathrm{d}$ intravenous infusion.

Contraindication Hypersensitivity to the drug. Hypersensitivity to the castor oil products.

Main Side effects Gastrointestinal upset, renal failure, seizure, neuropathy, hyperkalemia, and tremor.

Special points The blood trough levels may be used for monitoring and levels range from 5 to 20 $\mathrm{mg} / \mathrm{mL}$. May benefit refractory MG patients [32,33, Class III].

Cost/ cost effectiveness FK 506 therapy for 1 month costs $\$ 300$ to $\$ 350$.

Reboot of the immune system with G-CSF/ Cyclophosphamide

Lymphocytes and committed hematopoietic progenitors are rapidly killed by high-dose cyclophosphamide, because they express low levels of aldehyde dehydrogenase [34,35, Class III]. After the treatment, the hematopoietic stem cells proliferate, enhanced by administration of granulocyte colony stimulating factor (G-CSF), and reconstitute the immune system. In principal, the newly developing (or "rebooted") immune system may recognize the autoantigen as "self", thereby tolerating it.

Standard procedure A recent study used extremely high doses $(50 \mathrm{mg} / \mathrm{kg}$ per day intravenously for 4 days followed by G-CSF) in three refractory patients with MG who did not respond to conventional immunotherapeutic agents or could not tolerate their side effects. All three patients tolerated therapy and improved, allowing for a reduction in immune medications. AchR-Ab titers fell in two patients and anti-MuSK titers fell in the third $[36 \cdot$, Class III]. 


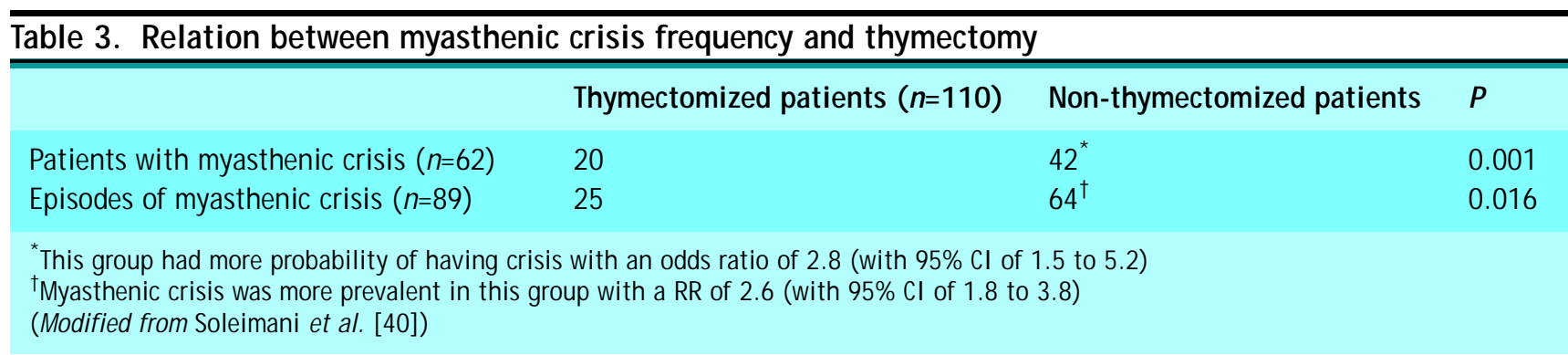

Special points This therapy is good for refractory patients who failed to respond to thymectomy, plasmapheresis, and conventional immunotherapeutic agents or can not tolerate their side effects.

Cost/ cost effectiveness Approximately $\$ 4000$ for the entire course of therapy.

Surgery

\section{Thymectomy}

- Thymectomy may be done for surgical removal of thymoma, and as a treatment for MG. Surgical removal of a thymoma is necessary because of the possibility of local tumor spread, although most thymomas are benign. In the absence of a tumor, the avail able evidence suggests that as many as $85 \%$ of patients experience improvement after thymectomy, primarily those with early onset MG who do not have anti-titin or anti-Ryanodine receptor antibodies [37, Class II]. A multicenter, international trial currently is being done [38, Class I ]. Of these, approximately 35\% achieved drug-free remission. In view of these potential benefits and of the negligible risk in skilled hands, thymectomy has gained widespread acceptance in thetreatment of MG. However, the improvement is typically delayed for months to years. It should not be attempted without minimizing patient weakness first. As a result, it is rarely used during hospital ization for crisis [39•, Class III]. Thymectomy seems to have a preventive role on rate and severity of future attadks [40, ClassI ] (Table 3).

\section{Prognosis}

- Median duration of hospitalization for crisis is 1 month. The patient usually spends half of this time intubated in the intensive care unit $[13,14,19,41$, Class III]. Approximately $25 \%$ of patients areextubated on hospital day 7 , $50 \%$ by hospital day 13 , and $75 \%$ by hospital day 31 . Risk factors for prolonged intubation (greater than 2 weeks) indude patient age older than 50 years, preintubation serum carbon dioxide levels above $30 \mathrm{mg} / \mathrm{dL}$, and highest VC of less than $25 \mathrm{~mL} / \mathrm{kg}$ during the first week of intubation [14, Class III]. In a study of 73 episodes of crisis [14, Class III], the risk of prolonged intubation was $0 \%$ in patients with none of these risk factors, $20 \%$ in those with onerisk factor, $50 \%$ in those with two risk factors, and $90 \%$ in those with three risk factors.

- Duration of intubation is an important predictor of functional outcomeafter crisis. In the same study [14, Class III], 77\% of patients intubated for more than 2 weeks had functional dependence at discharge compared with $36 \%$ of patients intubated for less than 2 weeks. The mortality rate during hospital ization for crisis has decreased from nearly $50 \%$ in the early 1960 s to between $3 \%$ and $10 \%$ today. With theincidence of crisis remaining stable over the past 30 years, this decrease in mortal ity rates reflects improvements in intensive care assessment and management of these patients. 


\section{References and Recommended Reading}

Papers of particular interest, published recently, have been highlighted as:

- Of importance

-. Of major importance

1. Fink ME: Treatment of critically ill patient with myasthenia gravis. In N eurology and N eurosurgical Intensive Care, Edition 3. Edited by Ropper AH. New York: Raven Press; 1993:351-362.

2. Wilks S: On cerebritis, hysteria, and bulbar paralysis, as illustrative of arrest of function of the cerebrospinal centers. Guy's H ospital Report 1877, 22:7-55.

3. Pascuzzi RM: The history of myasthenia gravis. $N$ eurol Clin 1994, 12:231-242.

4. Drachman DB: Myasthenia gravis. N Engl J M ed 1994, 330:1797.

5. Phillips II LH: The epidemiology of myasthenia gravis. N eurol Clin 1994, 12:263-271.

6. Qureshi Al, Choudhry MA, Mohammad Y, et al.: Respiratory failure as a first presentation of myasthenia gravis. M ed Sci M onitor 2004, 10:CR684-689.

7. Kubis KC, Danesh-Meyer HV, Savino PJ, Sergott RC: The Ice test versus the rest test in myasthenia gravis. 0 phthalmology 2000, 107:1995-1998.

8. Lertchavanakul A, Gamnerdsiri P, Hirunwiwatkul P: Ice test for ocular myasthenia gravis. J med Assoc Thai 2001, 84( suppl 1):S131-136.

9. Hoch W, McConvilleJ, Helms S, et al.: Auto-antibodies to the receptor tyrosine kinase musk in patients with myasthenia gravis without acetylcholine receptor antibodies. $N$ at $M$ ed 2001, 7:365-368.

10. Wilkins KB, Bulkley GB: Thymectomy in the integrated management of myasthenia gravis. Adv Surg 1999, 32:105-133.

11. Bedlack RS, Sanders DB: How to handle myasthenic crisis. Essential steps in patient care. Postgrad M ed 2000, 107:211-214.

12. Mayer SA: Intensive care of the myasthenic patient. N eurology 1997, 48:70-75S.

This is a nice review of the topic from an expert in this field.

13. Berrouschot J, Baumann I, Kalischewski P, et al.: Therapy of myasthenic crisis. Crit Care M ed 1997, 25:1228-1235.

14. Thomas CE, Mayer SA, Gungor Y, et al.: Myasthenic crisis: Clinical features, mortality, complications, and risk factors for prolonged intubation. N eurology 1997, 48:1253-1260.

15. Qureshi Al, Bhardwaj A, Ulatowski JA: Neurocritical care for the house officer. In ICU Care House O fficer Series. Edited by Helfaer M. Baltimore: Williams \& Wilkins; 1998:65,92.

This is a good review for the neurologists in training or those specialists not involved with neuromuscular care directly.

16. •Janjua N, Mayer SA: Myasthenic crisis. In Critical Care

$\mathrm{N}$ eurology and $\mathrm{N}$ eurosurgery, Edition 1. Edited by Suarez JI. New Jersey: Humana Press; 2004:469-480.

This book is by far one of the most comprehensive efforts on elaborate management issues pertai ning to neurocritical care This particular chapter is an exhaustive review of the topic with very focused and practical management aspects discussed by the experts in the field of neurocritical care

17. Younger DS, Raksadawan N: Therapy in neuromuscular disease. N eurol Clin 2001, 19:205-215.

18. Mayer SA, Thomas CE: Therapy of myasthenic crisis. Crit Care M ed 1998, 26:1136-1137.

This is another nice review on this topic.

19. London SF RS: Neuromuscular emergencies. In Emergent and U rgent N eurology. Edited by Weiner WJ. Philadel phia: JB Lippincott; 1992:59-78.

20. Rieder $P$, Louis $M$, Jolliet $P$, Chevrolet JC: The repeated measurement of vital capacity is a poor predictor of the need for mechanical ventilation in myasthenia gravis. Intensive Care M ed 1995, 21:663-668.

21. Tripathi M, Kaushik S, Dubey P: The effect of use of pyridostigmine and requirement of vecuronium in patients with myasthenia gravis. J Postgrad M ed 2003, 49:311-314; Discussion 314-315.

22. Q Qureshi Al, Choudhry MA, Akbar MS, et al.: Plasma exchange versus intravenous immunoglobulin treatment in myasthenic crisis. N eurology 1999, 52:629-632. There is a lack of major trials and evidence based treatment options for patients with myasthenia. This paper deals with a small number of patients but represents an excellent effort to address this important question.

23. - Gajdos P, Chevret S, Clair B, et al.: Clinical trial of plasma exchange and high-dose intravenous immunoglobulin in myasthenia gravis. Myasthenia gravis clinical study group. Ann N eurol 1997, 41:789-796.

This is another effort to address a very similar question represented in a relatively small subset of patients.

24. Stricker RB, Kwiatkowska BJ, Habis JA, Kiprov DD: Myasthenic crisis: Response to plasmapheresis following failure of intravenous gamma-globulin. Arch N eurol 1993, 50:837-840.

25. - Qureshi Al, Suri MF: Plasma exchange for treatment of myasthenia gravis: Pathophysiologic basis and clinical experience. Ther A pher 2000, 4:280-286.

This is a good review for plasma exchange option in myasthe nia gravis. The authors represent their own clinical experience in a practical and evidence-based approach.

26. Wolfe GI, Barohn RJ, Foster BM, et al.: Randomized, controlled trial of intravenous immunoglobulin in myasthenia gravis. M uscle N erve 2002, 26:549-552.

27. Confavreux C, Saddier P, Grimaud J, et al.: Risk of cancer from azathioprine therapy in multiple sclerosis: A case-control study. N eurology 1996, 46:1607-1612.

28. Meriggioli MN, Rowin J, Richman JG, Leurgans S: Mycophenolate mofetil for myasthenia gravis: a double-blind, placebo-controlled pilot study. Ann N Y Acad Sci 2003, 998:494-499.

29. Meriggioli MN, Rowin J: Single fiber EMG as an outcome measure in myasthenia gravis: results from a double-blind, placebo-controlled trial. J Clin N europhysiol 2003, 20:382-385. 
30. DeFeo LG, Schottlender J, Martelli NA, Molfino NA: Use of intravenous pulsed cyclophosphamide in severe, generalized myasthenia gravis. M uscle $N$ erve 2002, 26:31-36.

31. Takamori M, Motomura M, Kawaguchi N, et al.: Antiryanodine receptor antibodies and FK506 in myasthenia gravis. N eurology 2004, 62:1894-1896.

32. Utsugisawa K, Nagane $Y$, Yonezawa $\mathrm{H}$, et al.: Effects of fk506 on myasthenia gravis patients with high interleukin-2 productivity in peripheral blood mononuclear cells. M uscle N erve 2003, 27:245-248.

33. Evoli A, Di Schino C, Marsili F, Punzi C: Successful treatment of myasthenia gravis with tacrolimus. M uscle N erve 2002, 25:111-114.

34. Gordon MY, Goldman JM, Gordon-Smith EC: 4-hydroperoxycyclophosphamide inhibits proliferation by human granulocyte-macrophage colony-forming cells (GM-CFC) but spares more primitive progenitor cells. Leuk Res 1985, 9:1017-1021.

35. Jones RJ, Barber JP, Vala MS, et al.: Assessment of aldehyde dehydrogenase in viable cells. Blood 1995, 85:2742-2746.

36. - Drachman DB, Jones RJ, Brodsky RA: Treatment of refractory myasthenia: "rebooting" with high-dose cyclophosphamide. Ann N eurol 2003, 53:29-34.

This is an innovative, but unproven approach to treat refractory MG.
37. Romi F, Gilhus NE, VarhaugJE, et al.: Thymectomy and antimuscle antibodies in nonthymomatous myasthenia gravis. Ann N Y Acad Sci 2003, 998:481-490.

38. Wolfe GI, Kaminski HJ, Jaretzki III A, et al.: Development of a thymectomy trial in nonthymomatous myasthenia gravis patients receiving immunosuppressive therapy. Ann N Y Acad Sci 2003, 998:473-480.

39. Kissel JT, Franklin GM: Treatment of myasthenia gravis: A call to arms. N eurology 2000, 55:3-4.

In this paper there is good effort and an attempt on highlighting the importance of seriousness of this disease process and urgency of promoting better research on this topic both in the clinical and basic care setting.

40. Soleimani A, Moayyeri A, Akhondzadeh S, et al.: Frequency of myasthenic crisis in relation to thymectomy in generalized myasthenia gravis: A 17-year experience. BM C N eurol 2004, 4:12.

41. Cohen MS, Younger D: Aspects of the natural history of myasthenia gravis: Crisis and death. Ann N Y Acad Sci 1981, 377:670-677.

A good review of natural history of myasthenia gravis and myasthenic crisis. 